\title{
De la planeación a la apropiación: un estudio de caso en torno a las prácticas de lectura en una escuela pública de Lambayeque
}

\author{
Johanna Ramírez Fernández
}

Pontificia Universidad Católica del Perú

jramirezf@pucp.pe

Recibido: 15-05-2018

Aprobado: 09-01-2019 


\title{
De la planeación a la apropiación: un estudio de caso en torno a las prácticas de lectura en una escuela pública de Lambayeque
}

\section{Resumen}

En oposición al enfoque de implementación que señala que los diferentes actores educativos, en especial los maestros, ponen en práctica las políticas educativas siguiendo una visión lineal y vertical, numerosas investigaciones ofrecen evidencia de cómo estos se apropian de las macropolíticas en sus diferentes niveles de concreción curricular y las traducen y reinterpretan (Castanheira, Crawford, Dixon y Green, 2001; Unamuno, 2015; Zavala, 2012). A partir de la teoría de la actuación de las políticas educativas (Ball, Maguire y Braun, 2012) y de los Nuevos Estudios de Literacidad (Barton y Hamilton, 1998; Brice Heath, 1983; Zavala, 2002; Zavala, Niño Murcia y Ames, 2004), en este artículo, analizo cómo dos maestros de un colegio público de Lambayeque hacen política en torno a la enseñanza de la lectura, desde sus realidades cotidianas, y sus condiciones materiales y contextuales. Mientras que uno de ellos propone una enseñanza de la lectura para aprobar el examen de admisión, el otro decide utilizar la lectura para desarrollar una visión crítica de la realidad social. Asimismo, doy cuenta de que, en la construcción de estas diferentes maneras de actuar en torno a la enseñanza de la lectura, se articulan relaciones complejas entre los actores institucionales, los discursos en torno al lenguaje y a su enseñanza en la escuela, y las prácticas y artefactos letrados que dan sentido a lo que para ellos significa leer en los últimos años de la educación secundaria.

Palabras clave: actuación de las políticas, Nuevos Estudios de Literacidad, políticas educativas, Perú, lectura crítica, educación secundaria

\section{From planning to appropriation: A case study related to reading practices in a public school in Lambayeque}

\begin{abstract}
In contrast to the implementation approach for which social actors, especially teachers, put into practice educational policies in a linear and vertical way, many studies offer evidence of how these agents appropriate, translate and reinterpret macro policies in the different levels of curricular application (Castanheira et al., 2001; Unamuno, 2015; Zavala, 2012). In this article, I use the framework of Educational Policy Enactments (Ball, Maguire y Braun, 2012) and the New Literacy Studies (Barton y Hamilton, 1998; Brice Heath, 1983; Zavala, 2002; Zavala, Niño Murcia y Ames, 2004) to analyze the way in which two teachers from a public school in the city of Lambayeque do policy on teaching reading based on their everyday contexts and material conditions. While one of them teaches reading so that students approve the examination to get into the university, the other one uses reading to develop in their students a critical consciousness of social reality. In addition, I discuss how these literacy practices are connected to complex relationships among institutional social actors, discourses related to language and its teaching in school, and literacy artifacts that give sense to what reading means to them in the last years of secondary education.

32 I Keywords: policy enactment, New Literacy Studies, educational policies, Perú, Critical Literacy, Secondary Education
\end{abstract}




\section{Introducción}

Los estudios tradicionales sobre políticas educativas (Fishman, 1979; Young, 1999) se han realizado sobre la base de la dicotomía entre creación e implementación de las políticas. Desde esta óptica, los únicos actores que harían política son quienes las "crean", en el entendido de que son sujetos autorizados para promulgarlas. Estos enfoques entienden la política como un conjunto de leyes o directrices normativas que los actores educativos cumplen en todos los niveles curriculares a los que la política alcanza. Desde esta perspectiva, los maestros, por ejemplo, serán considerados solo como implementadores de lineamientos curriculares oficiales sobre los cuales no tienen ningún control o agencia (Cassels, 2013). A diferencia de estos estudios tradicionales, los enfoques críticos prestan atención a la puesta en práctica de las políticas educativas oficiales. Es decir, analizan la forma como los sujetos educativos se relacionan, integran y discuten con los lineamientos curriculares nacionales en los escenarios institucionales y pedagógicos en los que interactúan (Levinson, Sutton y Winstead, 2009). En este sentido, el foco está en comprender cómo hacen política los actores educativos, qué contradicciones y tensiones permean esta actuación, de qué recursos materiales disponen para la puesta en práctica de las políticas, cuáles son los discursos de poder que restringen su actuación, y qué sentidos y significados sostienen sus maneras de actuar en los escenarios educativos concretos (Ball, Maguire y Braun, 2012).

Por otro lado, las investigaciones en torno a la lectura se han centrado en analizar los procesos cognitivos involucrados en la comprensión lectora y en las estrategias que los estudiantes necesitan para aprender a leer con más eficacia (Inga, 2007; Pinzas, 2004; Pinzas, Chiuyare y Macavilca, 2008). Desde este marco, la lectura se ha asumido como una habilidad cognitiva y técnica, desvinculada de las condiciones discursivas, sociales y culturales en las que se lleva a cabo (Gee, 2005). Apartándose de esta perspectiva, los enfoques socioculturales apuestan por una visión de la lectura como práctica situada en actividades sociales concretas, articulada por determinados propósitos y mediada por ideologías (Barton y Hamilton, 2004).

En este artículo, analizo cómo dos maestros que dictan el mismo curso, Comunicación, en un colegio público de Lambayeque hacen política en torno a la enseñanza de la lectura desde sus realidades cotidianas y sus condiciones materiales y contextuales. La investigación demuestra que uno de ellos propone una enseñanza de la lectura para aprobar el examen de admisión y el otro decide utilizar la lectura para desarrollar una visión crítica de la realidad social. Este análisis evidencia, una vez más, que la relación entre lo que se plantea en los documentos curriculares institucionales y lo que se hace en las aulas no es directa, ni de arriba hacia abajo, ni de creación e implementación. Por el contrario, constituye una práctica constante de reinterpretación de significados y sentidos, sobre lo que los maestros y estudiantes creen que deben saber en torno a la lectura antes de culminar la educación secundaria (Unamuno, 2015). 
Este estudio se alinea con las crecientes investigaciones que analizan las prácticas de los actores educativos para comprender qué ocurre con las políticas oficiales en los microniveles curriculares. Su importancia reside en que da cuenta de qué políticas hacen los maestros en relación con la lectura, cómo hacen estas políticas, qué sentidos articulan sus decisiones pedagógicas en lo que respecta a la lectura en sus aulas, y cuál es la relación entre estas traducciones y las políticas educativas nacionales e institucionales. Asimismo, es relevante porque, en el Perú, a pesar de la importancia del fenómeno educativo, los estudios acerca de cómo se concretizan las políticas macrocurriculares en las aulas aún son escasos (González, Eguren, De Belaunde, 2014). Para el caso de la lectura en educación secundaria, no hay estudios que hayan realizado un análisis desde esta perspectiva.

\section{Marco teórico}

Los estudios sobre las políticas educativas han transitado desde una visión centrada en los documentos oficiales y en quienes los elaboraron hacia una mirada de las formas en las que los actores educativos se apropian de estos discursos. Las visiones más tradicionales sobre el estudio de las políticas educativas asumen que la implementación de estas sigue un proceso aislado, lineal y vertical (o top-down), es decir, de la "elaboración" de los responsables de su planeación a la "aplicación" de los "ejecutores" (Cassels, 2013). Estos estudios han tomado como focos de análisis de política los documentos que la oficialidad educativa promulga para comprender, por ejemplo, si estos funcionan efectivamente $o$ no, y qué factores influyen en su mal o buen funcionamiento (Levinson, Sutton y Winstead, 2009, p. 768). Para quienes defienden estos enfoques, los actores educativos involucrados en los diferentes niveles de concreción curricular son considerados solo "implementadores" de las decisiones curriculares planificadas por responsables que están por encima de ellos. De esta manera, estos enfoques ignoran e invisibilizan las formas particulares en las que los actores educativos se aproximan a las políticas en las diferentes instancias a las que esta alcanza (Cassels, 2013).

A diferencia de lo planteado por los enfoques tradicionales, los enfoques críticos, desde una perspectiva etnográfica, asumen que la puesta en marcha de las políticas educativas en los diferentes niveles curriculares implica un complejo proceso de negociaciones en el que los actores toman los elementos de la política nacional y los resignifican, traducen y reinterpretan en función de sus propios esquemas de interés, motivación y acción (Levinson y Sutton, 2001). Así, la política constituye un proceso que "se disputa diversa y repetidamente y/o se somete a una interpretación diferente, a medida que se promulga, de manera original y creativa dentro de las instituciones y aulas" (Ball, Maguire y Braun, 2012, pp. 2-3). Esta manera de entender la política enfatiza la agencia de los actores educativos en el proceso de apropiación y construcción de la política en todos los niveles curriculares a los que esta alcanza (Menken y García, 
2010). De este modo, cuando un docente decide enfatizar un tipo de contenido y prescindir de otro, seleccionar un tipo de evaluación en detrimento de otra u optar por una metodología dentro de varias posibilidades metodológicas, está haciendo política educativa y, asimismo, está ejerciendo poder.

Si bien este enfoque crítico enfatiza la agencia de los actores educativos en los procesos de apropiación, no deja de reconocer que estas traducciones siempre están restringidas por un marco de poder más amplio. Al respecto, Ball señala que existen luchas reales entre lo que se promulga y lo que los actores educativos interpretan; sin embargo, estas se establecen "dentro de un marco discursivo en movimiento que articula y restringe las posibilidades y probabilidades de interpretación y representación" (1993, p. 15). De esta manera, las opciones de los educadores pueden verse limitadas por lo que, en general, está permitido o no en la institución educativa, o lo que consideran "normal" o lo que se "ajusta al contexto". Es un hecho que la línea de poder no fluye linealmente desde quien elabora la política hasta las elecciones particulares del maestro en las aulas con sus estudiantes. Así,

la negociación en cada nivel institucional crea la oportunidad de reinterpretaciones y manipulación de políticas. Los educadores locales no están indefensos atrapados en el flujo y reflujo de las ideologías cambiantes en las políticas [educativas]: ayudan a desarrollar, mantener y cambiar ese flujo. (Cassels, 2013, p. 97)

Este artículo se enmarca en la segunda aproximación a la política educativa. El análisis que presento asume la política como un proceso complejo y multidimensional inscrito en relaciones de poder (Ball, 1993; Levinson, Sutton y Winstead, 2009). En el marco de restricciones discursivas más amplias, los directivos, los padres de familia, los profesores y los estudiantes negocian lo que significa para ellos la escuela básica, la formación en educación secundaria, el rol del profesor, el rol de la lectura. Esta negociación de significados se traduce en prácticas o actuaciones microcurriculares que extienden; contextualizan; y, en algunas ocasiones, retan las versiones oficiales de las políticas y los discursos hegemónicos en las que estas se inscriben. De esta manera, la apropiación de las políticas educativas constituye una práctica de poder que, dependiendo de las actuaciones y traducciones en las que los actores se comprometen, producirá determinados sujetos de política en los diferentes niveles curriculares (Ball, 1993; Fairclough, 1992).

En esta misma línea, para analizar lo que los maestros y los estudiantes hacen con los textos en las aulas, utilizaré los aportes de los Nuevos Estudios de Literacidad. Estos enfoques constituyen una mirada crítica a lo que Street (1984) denominó el modelo "autónomo" de literacidad, el cual supone que la lectura y la escritura son herramientas procedimentales que desarrollan por sí mismas los procesos cognitivos "superiores" y propician el uso descontextualizado del lenguaje. Frente a una visión que concede a lo letrado un poder 
cognitivo independiente del contexto, Street (1984) propone el modelo "ideológico" de literacidad, que plantea la necesidad de mirar los usos de lectura y escritura situados en el contexto sociocultural en el que se llevan a cabo. Esta contextualización permite explicar estos usos como prácticas sociales mediadas e imbricadas en relaciones de poder, asociadas con un gran número de factores, entre los que están las condiciones políticas y económicas, la estructura social y la ideología (Gee, 2005; Street, 2004). De esta manera, desde una perspectiva etnográfica, la atención no solo se centra en los textos en sí mismos, sino, fundamentalmente, en las actividades sociales que los sujetos realizan en torno a esos textos, a los pensamientos que sostienen esos modos particulares de interactuar, y a los significados e importancia que los sujetos le otorgan a lo letrado en su vida cotidiana en cualquier esfera social (Barton y Hamilton, 1998). En función de este análisis, utilizaré dos categorías teóricas centrales para comprender la forma en la que los maestros y estudiantes se aproximan a los textos: evento letrado y práctica letrada.

Un evento letrado, según Barton y Hamilton (1998), es una actividad social en la que lo letrado cumple un rol. Son eventos letrados en el ámbito educativo, por ejemplo, la resolución de un examen, el registro de asistencia, el momento de aplicación de una ficha de evaluación, la lectura de un texto con fines de análisis, etc. En nuestras actividades cotidianas, estamos constantemente inmersos en eventos letrados como la lectura de un periódico, el llenado de un formulario para una cita médica, la lectura de la pantalla electrónica para abordar un bus o avión, o un croquis para ubicar una dirección. Es preciso señalar que la noción de evento acentúa la naturaleza situacional de la literacidad. Es decir, no leemos en abstracto, siempre leemos en el marco de una actividad social concreta y con fines específicos.

Por otro lado, las prácticas letradas son las maneras aceptadas y recurrentes de leer y escribir en los eventos letrados específicos. La categoría práctica letrada reúne dos dimensiones en su conceptualización: el hacer, que es la dimensión externa observable, y el porqué de ese hacer, que abarca creencias, valores, intereses. Es decir, integra las maneras culturales como proceden los sujetos con los textos y los valores, actitudes, sentimientos y relaciones sociales que sostienen esas maneras de hacer (Street, 2004). Barton y Hamilton (1998) señalan que "la noción de práctica letrada ofrece una manera poderosa de conceptualizar el enlace entre las actividades de lectura y escritura, y las estructuras sociales en las cuales se encuentran imbricadas y las cuales ayudan a delinear" (p. 112). De este modo, las maneras particulares de leer y escribir no se reducen a dar cuenta de las capacidades cognitivas de los sujetos que leen o de la dificultad de los textos a los que estos acceden. Por el contrario, involucran una dimensión social que vincula propósitos sociales específicos, articula modos particulares de entender la realidad social y exhibe procesos de negociación por construcción de identidades en el marco de determinadas relaciones sociales (Barton y Hamilton, 2004; Street, 2004). 


\section{Metodología}

La escuela donde llevé a cabo esta investigación se ubica en el distrito de $\mathrm{Pu}$ calá, provincia de Chiclayo, Región Lambayeque. Brinda el servicio educativo en el Nivel Secundario de Menores, en la variante de Ciencias y Humanidades, para alumnos y alumnas procedentes del cercado de Pucalá, de sus caseríos aledaños y del distrito de Pátapo. Los estudiantes de esta institución educativa provienen, en su mayoría, de hogares cuyos padres y/o tutores trabajan en la ex cooperativa agraria, hoy Empresa Agro Industrial "Pucalá". Esta es una institución educativa mixta. No obstante, en las aulas, los estudiantes están separados en grupos de hombres y de mujeres. En las secciones que observé, tanto el profesor como la profesora trabajan con grupos de mujeres.

Para comprender la forma en la que los actores educativos construyen política en torno a la lectura en educación secundaria, utilicé una perspectiva etnográfica: he observado interacciones dentro y fuera del aula, he analizado los materiales de enseñanza-aprendizaje, y he realizado entrevistas a los actores educativos: subdirector académico, maestros y estudiantes observados, padres de familia. A nivel de las interacciones en el aula, registré y grabé en video las prácticas pedagógicas de dos maestros, una de cuarto grado y el otro de quinto grado de educación secundaria, ambos de las secciones D. Además de la observación de las prácticas pedagógicas, registré los materiales didácticos que utilizaban los maestros y sus estudiantes. Llevé a cabo la observación etnográfica desde el mes de marzo hasta el mes de mayo de 2013, según la programación del horario institucional para las secciones señaladas.

Para ahondar en las representaciones e identidades que construyen y recrean los diferentes agentes del proceso educativo en torno a las prácticas de lectura, realicé entrevistas semiestructuradas. En primer lugar, entrevisté al subdirector académico de la institución educativa en dos oportunidades para conocer la "voz oficial institucional" con respecto al valor de la lectura, y a cómo esta era entendida y enseñada en la institución. En segundo lugar, entrevisté a los profesores de aula, cuyas sesiones de aprendizaje observé. Estas entrevistas tuvieron como objetivo recoger sus percepciones sobre cómo entienden la lectura, por qué seleccionan determinados textos, por qué privilegian determinadas prácticas en su enseñanza y qué importancia le asignan a esta en la formación escolar básica. Asimismo, entrevisté a las estudiantes de $4^{\circ}$ grado D para recoger sus percepciones y valoraciones en relación con la forma como se desarrollan las prácticas en torno a la lectura, y para conocer la importancia que le asignan. Finalmente, decidí entrevistar a dos grupos de cinco madres y padres de familia para acercarme a las representaciones que construyen y privilegian en lo que respecta al valor de la lectura en la escuela y a la escolarización de sus hijos en general. Estas entrevistas se llevaron a cabo al principio y al final de la etapa de observación. El tiempo promedio de las entrevistas oscila entre 35 y 55 minutos. Todos estos datos fueron procesados a través de la lectura analítico-interpretativa. 


\section{Análisis}

En esta sección, explicaré cómo los actores educativos se han apropiado de la política nacional e institucional. En un primer momento, analizaré la traducción que han realizado del Diseño Curricular Nacional (DCN) en las programaciones curriculares de cuarto y quinto año de educación secundaria del curso de Comunicación. En un segundo momento, analizaré cómo dos maestros se apropian, asimismo, de esta primera interpretación en las interacciones pedagógicas con sus estudiantes.

\section{Primera apropiación de las políticas: las programaciones curriculares insti- tucionales}

Los actores educativos, aprovechando el espacio de diversificación curricular que ofrece el Diseño Curricular Nacional (DCN), incorporaron en sus programaciones curriculares de cuarto y quinto grado de secundaria contenidos que se evalúan en el examen de admisión para ingresar a la universidad, específicamente, contenidos relacionados con razonamiento verbal y razonamiento matemático. Esta apropiación (Levinson y Sutton, 2001) particular de la política educativa nacional se enmarca en un contexto externo (Ball, Maguire y Braun, 2012) caracterizado por los discursos mercantilistas de la educación, la ideología del logro (Torres, 2002, 2010) y la percepción creciente de la escuela pública como una institución carente de utilidad en comparación con la escuela privada, que se percibe como de mejor calidad (Cuenca, 2013; Torres, 2010). En el análisis, se evidenciará que el contexto externo, entendido como un conjunto de discursos globales y locales acerca del fenómeno educativo, ejerce presión sobre el currículo de la institución educativa.

Para los padres de familia, el acceso a la educación universitaria constituye un mecanismo que puede garantizar la movilidad social de sus familias. Esta representación se instala en el mito de la educación para el progreso que, según Tubino (2007), en la actualidad, se ha trasladado desde la escuela básica a la educación superior: la universidad se percibe como "el medio de abrirse futuro promisorio en medio de una sociedad de restringidas oportunidades" (p. 117). La vigencia de este mito refleja la importancia de la educación universitaria en el imaginario social de muchas familias peruanas, aun cuando la educación superior como mecanismo igualador de oportunidades es todavía limitado (Benavides y Etesse, 2012, p. 78). En ese sentido, para acceder a este bien social, sus hijos deben superar el examen de admisión. Ello implica para los padres, en términos estratégicos, buscar mecanismos que permitan que sus hijos ingresen a la universidad.

Tradicionalmente, en nuestro país, a partir de la masificación del acceso a la educación universitaria y de la promulgación de la Ley de Promoción de la Inversión en Educación, las academias preuniversitarias son y han sido los espacios legítimos en los que se preparan a los estudiantes para postular a las 
universidades. Sin embargo, hoy en día, no solo las academias preuniversitarias se encargan de esta tarea, sino que, progresivamente, los colegios - en su mayoría, privados - han asimilado el programa escolar a una lógica preuniversitaria (Hurtado, 2006). Así, para preparar a sus hijos para el examen de admisión, los padres acuden a las academias preuniversitarias o a los colegios privados preuniversitarios. En ambos casos, preparar a sus hijos para postular a la universidad implicaría un gasto económico adicional en el que la familia debe invertir para conseguir beneficios a futuro. La diferencia entre estas dos modalidades está en que en los colegios preuniversitarios los preparan desde antes de terminar la educación secundaria.

Si bien la preocupación de los padres por el futuro universitario de sus hijos no es un fenómeno nuevo, en la institución educativa en la que llevé a cabo la investigación, llama la atención que esta preocupación se traduzca en un nuevo encargo social para la escuela pública: esta debe preparar a los estudiantes para el examen de admisión. De esta manera, el pedido/exigencia de los padres, en tanto actores sociales estratégicos (Ball, 2003), tiene un rol primordial en el reordenamiento de las programaciones curriculares de cuarto y quinto grado de la institución educativa. A continuación, cito la voz del subdirector para evidenciar la demanda:

Entonces, el papá pide que "no, como en el otro colegio" [se refiere al colegio particular]. Entonces, te hacen la comparación: "allá le dan dos horas o tres horas para Razonamiento Verbal [contenido que será evaluado en el examen de admisión], y queremos que sea asî". Buscamos satisfacer sus necesidades, en cierta medida, no en todo porque hay muchas cosas que nos piden, pero tampoco está a nuestro alcance. Si estamos enseñando Razonamiento Verbal, lo estamos adecuando, pero tampoco podemos dar más de lo que no nos pide el Ministerio, porque, quiera o no, también entonces dicen que se dedican más al Razonamiento Verbal. No es un centro preuniversitario y algunos papás qué hacen como no hay, según ellos la exigencia, sacan y llevan a sus hijos al colegio particular preuniversitario. (Subdirector académico de la institución educativa [el énfasis es mío])

Desde la voz del subdirector, el padre de familia es un agente central en la construcción de una nueva subjetividad de la escuela: esta debe proveerle productos que se alineen con sus necesidades particulares. El uso de procesos materiales o verbos de acción (Halliday, 1985) - "el papá pide", "te hacen la comparación", "sacan y llevan a sus hijos" - y de aseveraciones categóricas ("queremos que sea asi”) a través del habla reportada nos permite reconocer a un padre que demanda a la escuela contenidos para obtener un beneficio concreto y que, ante el incumplimiento de esto, toma decisiones en función de lo que le conviene: "saca y lleva a sus hijos al colegio particular preuniversitario". Esta identidad se ve reforzada por la posición que asume el subdirector en relación con las deman- 
das del padre de familia: "buscamos satisfacer sus necesidades". Si bien, desde su percepción, "satisfacer las «necesidades» del padre" constituye un imperativo que la escuela no debe desatender, tampoco "puede dar más de lo que pide el Ministerio". De esta manera, se percibe un conflicto entre lo que las políticas nacionales promulgan y las libertades que tienen las instituciones específicas para atender las demandas, en este caso, para incorporar lo solicitado por los padres de familia. Esta tensión revela que la apropiación de las políticas educativas por parte de los actores siempre se instala en un marco o discurso mayor que las restringe (Ball, 1993). Si bien la línea de poder no fluye linealmente entre lo que plantea el DCN y los procesos de apropiación de los actores educativos, estos se enmarcan dentro de lo que las políticas nacionales contemplan.

Para reforzar esta visión de los padres como agentes en las decisiones escolares, incluyo la voz de una de las profesoras entrevistadas, que reporta lo que los demás miembros de la comunidad educativa le han comentado con respecto a la "adecuación" de las programaciones curriculares:

Cuando me di cuenta de que la malla curricular era diferente a la que pedía el Ministerio, entonces, es ahí donde ellos [se refiere a los demás miembros de la institución educativa] me dijeron: "no, nosotros como nos dimos cuenta de que empezaron - hay creo dos colegios particulares acá- los padres empezaron a sacar a los chicos de acá del colegio nacional, empezaron a sacarlos a los particulares, porque decían que nosotros no enseñábamos como nivel pre. Entonces, nosotros tratamos de adecuar nuestra programación de acuerdo a lo que pedía el prospecto de la universidad". Es por eso que, en nuestra programación, hemos agregado Razonamiento Verbal. (Profesora de cuarto año [el énfasis es mío])

A partir de este extracto y del anterior, se refuerza la identidad del padre agente que ejerce su poder de elección escolar en función de las necesidades e intereses que persigue: deja el colegio nacional y opta por un colegio particular preuniversitario. En este sentido, la actuación del padre constituye una estrategia de negociación sobre la política de elección de escuela en función de las expectativas sobre los beneficios que obtendrá de esta elección (Sanz, 2015). Esta actuación se articula con una dinámica de mercado en la que los clientes eligen aquellos productos que les serán rentables y útiles en términos de adquisición de capital. Según Torres (2002), en el terreno educativo, "un buen producto" será aquel que permita obtener un beneficio inmediato; por el contrario, aquello que no implique una retribución personal/individual será desechado, cambiado o eliminado.

En esta dinámica mercantilista, para los padres de familia, los colegios particulares constituyen una mejor opción. Dicha percepción obliga a la escuela pública a modificar sus sentidos sociales y a alinearse a una lógica competitiva con la escuela privada en un intento por tener sentido como institución (Torres, 40 I 2002). En el siguiente extracto, un comentario de la profesora revela el sentir de 
los estudiantes con respecto a las prácticas de lectura en Razonamiento Verbal que se han incorporado en las programaciones curriculares de cuarto año: "Lo que me están enseñando no es por las puras; o sea, yo voy a seguir aprendiendo porque quizás voy a dar en el futuro un examen de admisión y voy a resolverlo bien. Entonces, me va a servir" (profesora de cuarto año [el énfasis es mío]).

Desde la voz de la profesora, se infiere que, para los estudiantes, la escuela adquiere validez en tanto permite obtener un resultado: resolver un examen de admisión. De lo contrario, la escuela se percibe como una institución carente de utilidad. De esta manera, la lógica de los resultados reconfigura el sentido de la escuela pública. Al respecto, el subdirector expresa la presión constante a la que es sometida su institución en función de los resultados obtenidos en los exámenes de admisión, en comparación con los resultados que obtienen los colegios particulares de la zona.

Acá hay dos colegios que son particulares y todos los años nos comparan: “¿cuántos ingresaron a la universidad de tal colegio y a qué carreras?" incluso. Por ejemplo, del colegio particular, este año ha ingresado una alumna entre los diez primeros puestos; ha ingresado a Medicina y ha sido el boom y otras carreras más. De acá [se refiere a su institución], han ingresado a la universidad, pero no a Medicina, pues. Es otro de los rótulos que te ponen en la frente: porque ingresaste, ya eres lo máximo. ¿Quién te pone ese rótulo? La sociedad misma ¿no? (Subdirector académico de la institución educativa)

Esta dinámica comparativa en la que los colegios particulares van ganando terreno, porque, según la percepción de la sociedad, obtienen mejores resultados debilita a la escuela pública como institución, en tanto esta termina reemplazando su tarea formativa por una tarea instrumental. Por ejemplo, lleva a que en la escuela se desarrolle una instrumentalización de la lectura con la finalidad de que los estudiantes memoricen los contenidos que serán evaluados en los exámenes de admisión. Este desplazamiento de sentido en torno al nuevo encargo social que debe cumplir la escuela hoy se articula con otros discursos relacionados con la medición de la calidad educativa y la mercantilización de la educación (Torres, 2010). La visión de la escuela como una institución que no tiene valor más allá de los resultados que los estudiantes puedan obtener de ella es peligrosa, en la medida que refuerza el discurso de los defensores de la mercantilización de la educación, quienes atacan la escuela pública alegando que no ha cumplido con las promesas de igualdad para todos los ciudadanos, por lo que debería dejar de existir como institución (Apple, 2002).

Como hemos podido ver, el marco o contexto externo ha desempeñado un papel fundamental en el primer nivel de apropiación de la política nacional. Las expectativas de los padres respecto a la función social de los estudios universitarios y la percepción de los colegios particulares como instituciones educativas de mejor calidad han permeado la forma como los actores educativos se apropian de la política educativa nacional y le dan un nuevo sentido a la 
escuela. En los siguientes apartados, analizaré un segundo nivel de apropiación de las políticas: las prácticas pedagógicas.

\section{Segunda apropiación de las políticas: las prácticas pedagógicas}

A partir de la perspectiva crítica de las políticas educativas y desde los Nuevos Estudios de Literacidad, en este apartado, analizaré como dos maestros - una de cuarto y otro de quinto grado de educación secundaria- hacen política y qué política construyen en torno a la lectura. Para ello, responderé las siguientes preguntas: ¿qué prácticas letradas despliegan los maestros en la interacción con sus estudiantes?, ¿qué sentidos se negocian en estas prácticas y sobre la base de qué creencias?, ¿de qué manera se articulan esas prácticas letradas con los sentidos esbozados en las políticas nacionales e institucionales?

\section{- Leer en la escuela para aprobar el examen de admisión}

En esta sección, analizaré las prácticas letradas en las que se comprometen la maestra junto con sus estudiantes. En el tiempo que observé, la maestra desarrolló los contenidos que la Programación Curricular de cuarto grado denomina Razonamiento Verbal: sinónimos, antónimos, analogías. El propósito en esas sesiones fue aprender para aprobar el examen de admisión, es decir, memorizar los significados de un grupo de palabras que son evaluadas recurrentemente en estos exámenes. A diferencia de la enseñanza generalizada del léxico, en la que se les ofrece a las estudiantes listas de palabras y se les pide que busquen directamente los significados en el diccionario, la profesora decidió enseñar el léxico de manera más creativa. En ese sentido, seleccionó textos como canciones o cuentos en los que se habían cambiado las palabras originales por palabras que ella y sus estudiantes denominan "desconocidas". Esto lo hace porque, desde su perspectiva, "si presentas las palabras en el contexto, se hace más fácil y más rápido de aprender, si es que tú quieres que te lo aprendan como para tipo examen de admisión". Ello lleva a que, en las sesiones de aprendizaje, el texto se convierta en un pretexto para la memorización del léxico con mayor facilidad y rapidez, lo cual propicia una instrumentalización de la lectura. Durante los momentos de lectura, la maestra y sus estudiantes se comprometieron en dos prácticas letradas recurrentes: reemplazar palabras en los textos mencionados e interactuar en torno a los textos a través de consignas de tono instructivo-procedimental.

A continuación, presento los versos de las canciones utilizadas como material didáctico ${ }^{1}$ en las sesiones de aprendizaje. En esta actividad, las estudiantes debían reemplazar las palabras resaltadas por sus significados.

1. Los materiales didácticos seleccionados forman parte del autoinstructivo Nemotecnia de memorización Tóser 22, cuyo autor es el docente Tomás Serquén Montehermoso. Desde la voz de la profesora "es un método práctico, interesante e innovador que te ayuda y ayuda a participar al alumno. [...] el contexto te dice mucho, ¿no? Gracias al contexto de la palabra en una lectura, te permite aprenderla más rápido". 


\title{
Ejemplo 1
}

Voy a pedirte

de hinojos

que regreses, junto a mí

porque soy de ti y te quiero como antes mucho más (Bis).

\author{
Ejemplo 2 \\ y yo estoy aquí \\ beodo y orate \\ y mi corazón mentecato \\ siempre fulgurará \\ y yo te amaré \\ perpetuamente.
}

Los versos originales de estas dos canciones son populares y son conocidos por las estudiantes, quienes, a medida que resuelven el ejercicio, los corean. Este saber previo les permite reemplazar con mucha facilidad las palabras resaltadas y se asombran, en algunas oportunidades, de los significados de estas: "ah, hinojos es rodillas". Asimismo, entre ellas, bromean y utilizan estas nuevas palabras para ponerse algún apodo o hacer referencia a algún pariente suyo en tono sarcástico: "el beodo de tu primo perdió el trabajo". En efecto, como se puede observar, por el involucramiento y la participación de las estudiantes, esta es una manera muy creativa y significativa de aprender para ellas, de acuerdo con el propósito que, en este contexto, la maestra y las estudiantes persiguen. Sin embargo, las prácticas de lectura en la escuela no deben limitarse a la memorización de un conjunto de palabras aisladas, sino que deben promover, además, una aproximación crítica a los textos (Zárate 2015).

Para seguir dando evidencia de esta práctica letrada, a continuación, presento el extracto de un cuento ${ }^{2}$ que utilizaron en clase.

Cuando alboreaba un nuevo día un pueblito situado a una distancia muy longincua, catorce kilómetros al este de Chiclayo se encuentra Tumán, tierra de clima caniculoso, sobre todo si la estación del estío es la que se encuentra de turno generando tedio a muchos pobladores que no titubean en ningún instante en acudir a las gélidas aguas de un río llamado "La Kalerita", solo tomas un carro y raudamente te conduce al lugar mencionado para que te bañes en sus diáfanas aguas que permiten visualizar los pececillos que esperan impacientemente el pipiripao perfecto que algunas personas suelen tirar al río (arroz cocido, migajas de pan, etc.) solo con el único propósito de ver como la carpanta de esas

2. Este cuento, utilizado como material didáctico en la sesión de aprendizaje, forma parte del autoinstructivo Nemotecnia de memorización Tóser 22, cuyo autor es el docente Tomás Serquén Montehermoso. 
diminutas criaturas hacen presenciar un lindo espectáculo, los personajes más liliputienses son los que terminan aplaudiendo mientras sus padres observan de manera absorta la rapidez de los peces por devorar algo que cae al río, ya sea porque alguien lo arrojó o porque de manera fortuita ha caído. Allí en ese mismo lugar observé a una linda niñita de ojos brunos, labios bermejos y pelo cimbreado, mientras el viento jugaba con su blonda cabellera, era la dulce inspiración que muchos vates desearían conocerla manceba, para inspirarse de su beldad pero solo tenía escasos cinco años, todos los domingos solía ir a aquel lugar para encontrarse con Serafín, un pez muy guapo y juguetón, era el más hercúleo de todos los que allí vivían, cada vez que Amandita lanzaba un bocado el pececillo tomaba fuerzas para poder alcanzarlo, ella se divertía mucho, mientras sus tersas manos acariciaban a Serafín.

En esta actividad, la maestra busca que las palabras que anteceden o preceden a las palabras subrayadas ayuden a las estudiantes a "identificar" los significados. De esta manera, se quiere lograr que las estudiantes se independicen del diccionario y puedan inferir con facilidad, a partir del contexto lingüístico, los significados de las palabras desconocidas durante el examen de admisión. Este ejercicio es interesante porque, a diferencia de la actividad anterior, las estudiantes no pudieron inferir con facilidad los significados de las palabras subrayadas, debido a que el texto ofrecido carece de un contexto y, además, ofrece varias posibilidades de inferencia que están relacionadas o que requieren de la experiencia inmediata de las lectoras: “¿brunos será grandes?”. Por este motivo, ellas prefieren ir directamente a su diccionario de sinónimos y antónimos argumentando que, desde su percepción, trabajar desde el contexto lingüístico "demora mucho". En ambas actividades, se observa que la lectura de los textos -cuento y canción- se reduce a la identificación y reemplazo de las palabras. En ese marco, no hay espacio para profundizar en las relaciones entre el uso del léxico y quién produce el texto, a quién va dirigido, con qué propósito comunicativo, en qué género discursivo, etc.

Otra de las prácticas letradas recurrentes, en el momento de lectura de los textos, es ofrecer consignas en tono instructivo-procedimental. A continuación, presento un extracto de la interacción entre la maestra y sus estudiantes antes de la lectura de un cuento.

1. Profesora: Aver,laindicación, atentas(shhhiiit) [hacecallaralasestudiantes]. A ver, vamos a trabajar de la siguiente manera, escuchen las indicaciones para que luego no tengan problemas ¿ya? A ver atrás, silencio, Ana, ¿ya? [...] Entonces, vamos a trabajar sinónimos, aún todavía no vamos a trabajar tipos de sinónimos (shhhiiit), de ejercicios de sinónimos; eso viene después. Primero, tratamos deubicarlosen un contextodeterminado.[...] La indicación acá sería, primero, darle una lectura atenta a todo el texto y luego ya identificar aquellas palabras ¿no? que son 
desconocidas, quizáspara ustedes, y con ayuda desu diccionario, esta vez, van a ir ¿no? completando y cambiando las palabras.

9. Estudiante: profesora, ¿usted ya ha subrayado la palabra?

10. Profesora: sí, ya están en negrita ¿isí? De repente si es que existe otra que quizás ustedes desconozcan también aprovéchenlo y usen su diccionario.

13. Estudiante: ¿qué diccionario?

14. Profesora: diccionario de sinónimos y antónimos. Aunque también un diccionario normal podría ayudarnos [...]. (Interacción profesora-estudiantes [el énfasis es mío])

Un aspecto que llama la atención en esta interacción es que las indicaciones para leer el cuento se brindan a modo de pasos consecutivos y ordenados que enfatizan tareas procedimentales como "darle una lectura atenta al texto", "identificar palabras". El uso de los conectores de orden - como "primero" y "luego"- y de los imperativos ("escuchen", "silencio") reducen la posibilidad de que las estudiantes problematicen la consigna o cuestionen la tarea que se les está encargando desarrollar. Sacristan (2013) señala que, en contextos en los que se educa con el propósito de evaluar un logro puntual, los maestros tienden a proporcionar instrucciones precisas y preguntas que evitan las explicaciones, cuestionamientos o repreguntas por parte de los estudiantes. Si bien, en esta interacción, las estudiantes utilizan su turno de intervención para preguntar, sus preguntas no plantean un cuestionamiento sobre la forma de leer. Por el contrario, solicitan información que les permita resolver la tarea encargada: ¿usted ha subrayado la palabra?, ¿en qué diccionario?, ¿se puede hacer en una hoja? Estas preguntas apuntan a aspectos que ellas podrían resolver por iniciativa propia y, sin embargo, recurren a la profesora. Esta dinámica interactiva se repite en todas las sesiones en las que el objetivo es leer para memorizar palabras que serán evaluadas en el examen.

Aun cuando ambas actividades contribuyen a lograr el propósito de la sesión, que es memorizar palabras para rendir un examen de admisión, es necesario mirar qué otras prácticas en torno al léxico se dejan de lado por perseguir este objetivo en la escuela. Por ejemplo, sería importante enseñar las palabras aprendidas como parte de situaciones comunicativas específicas. Esto permitiría que las estudiantes puedan analizar los contextos en los que estas palabras son socialmente pertinentes, con qué participantes y en qué propósitos sociales, y dentro de qué géneros discursivos. Abstraer el léxico de sus contextos de uso impide que las estudiantes puedan cuestionar las relaciones de poder en las que se inscriben los usos del lenguaje. Esta reflexión es importante, porque, cuando les pregunté a las estudiantes qué importancia tenía para ellas el aprendizaje de léxico, una de ellas, por ejemplo, señaló lo siguiente:

Nos ayuda a enriquecer nuestro vocabulario, ya que, a través de los sinónimos y antónimos, podemos conversar con una persona, por ejemplo, 
más culta. [...] Si sabemos nuevas palabras y sus significados, entonces, eso podemos acoplarlo a nuestro idioma y hablarlo con otras personas y se ve bonito, porque se nota que es un idioma más culto, sin tanta jerga o sin errar al hablar. (Estudiante de cuarto año [el énfasis es mío])

Desde el discurso de la estudiante, se puede observar que hay un ideal aspiracional que se sostiene en el mejoramiento del vocabulario. Desde su perspectiva, dejar atrás sus formas de hablar con "jergas" y "errar al hablar" implica poder acceder a otro grupo social de mayor prestigio, cuyo idioma es "más culto" y se "ve bonito". Así, "enriquecer el vocabulario" constituye una práctica de higiene verbal, que tiene como propósito "mejorar" o "limpiar" el lenguaje (Cameron, 1995), y se apoya en la creencia no cuestionada de que hay formas correctas e incorrectas de hablar adscritas indefectiblemente a determinados grupos en la sociedad. Asimismo, es interesante notar que, para esta estudiante, hablar "mejor" está asociado con el uso de un vocabulario rebuscado. Esta idea es compartida con el hallazgo de Mesía (2014), quien, en un estudio comparativo sobre las ideologías lingüísticas entre un colegio público y otro privado en Lima, señala que, para los estudiantes del colegio estatal, un indicador de "hablar bien" es conocer el significado de las palabras y contar con un vocabulario "culto" (p. 31). Dicha percepción se diferencia de la identificada en los estudiantes del colegio particular para quienes, además del léxico, "es necesario un conjunto de «recetas» $\mathrm{y}$ «técnicas» gramaticales que van a resultar en un "producto» bien elaborado" (pp. 31-32).

Ambas prácticas letradas analizadas en los párrafos anteriores dan cuenta de cómo la maestra y sus estudiantes se apropian de la política institucional y construyen una política en torno a la lectura desde una perspectiva instrumental. Esta apropiación particular de la política, como toda práctica de poder, produce determinados sujetos de política (Ball, 2008). En el caso analizado, reemplazar palabras, y seguir procedimientos que no promueven la crítica y la reflexión sobre lo que se aprende refuerza una identidad del estudiante como sujeto pasivo en la construcción de sus aprendizajes. De este modo, si en las sesiones de aprendizaje se insiste constantemente en repetir, memorizar y seguir instrucciones sin espacio para dialogar, las habilidades que las estudiantes desarrollen estarán íntimamente relacionadas con estas prácticas (Scribner y Cole, 1981). Probablemente, las estudiantes podrán resolver mecánicamente las preguntas en el examen de admisión. Sin embargo, ¿de qué manera dialogan estas formas de leer con las que privilegia la universidad? En el espacio universitario, ellas se enfrentarán, por ejemplo, a textos con una perspectiva más distante (expresiones impersonales), una sintaxis más compleja (oraciones de relativo, participio infinitivo) (Schleppegrell, 2005). Además, tendrán que leer en constante diálogo e interacción con otros textos para analizar, interpretar, criticar etc. En ese sentido, ¿en qué medida repetir, memorizar y seguir instrucciones contribuyen a formar un estudiante crítico y reflexivo de su entorno social? 


\section{- Leer en la escuela para asumir una posición crítica de la realidad social}

En esta sección, analizaré las prácticas letradas del profesor de quinto grado con sus estudiantes. Para el profesor, es importante que la escuela secundaria forme estudiantes que "se den cuenta de lo que sucede a su alrededor y que no sean simplemente receptoras de lo que acontece, sino que ellas tomen también su posición de acuerdo a cómo pueden ver las cosas". Para lograr este propósito, a partir de una aproximación crítica de la pedagogía de la lectura, el profesor selecciona textos con temáticas vinculadas a la realidad cotidiana de las estudiantes, por ejemplo, noticias, historias vivenciales, publicidad etc. A continuación, explicaré dos de las prácticas letradas en las que el maestro y las estudiantes se comprometen habitualmente: análisis de los textos como una construcción parcial e interesada de la realidad social, y la interrogación constante.

Según Cassany y Castellà (2010), una de las principales tareas, en el marco de una perspectiva crítica de la lectura, es desestabilizar la visión de los textos como verdades absolutas y neutrales. En esta línea, cuando las estudiantes leen los textos, el profesor las orienta a entender el texto como un discurso que incluye y excluye ciertas voces, que proyecta una versión del tema a tratar, y que va dirigido a un lector específico. El extracto que presento a continuación es parte de la interacción entre el maestro y las estudiantes después de leer una nota periodística, cuyo título era "La madre de hoy usa las redes sociales y odia las labores del hogar". Esta nota fue publicada en un diario de circulación nacional en el Día de la Madre. En el cuerpo de la nota, el diario ofrecía una infografía con datos que configuraban el "perfil de la madre moderna". Veamos el extracto:

1. Profesor: Qué tema trata la noticia, qué tema trata el texto, de qué nos habla el texto.

2. Ana: de las mamás modernas (en voz baja)

3. Profesor: de las mamás modernas

4. Ángela: ya no quieren que le regalen cosas para su hogar, sino para ellas, que las puedan utilizar ellas.

6. Profesor: para ellas. La mamá moderna y ¿cuál es la característica que presentan aquí para la mamá moderna?

8. Ana: no les gusta encargarse de las labores domésticas

9. Profesor: ¿la mamá moderna está de la mano con?

10. Isabel: ¿los electrodomésticos?

11. Gabriela: con la tecnología

12. Profesor: ¿los electrodomésticos? con la tecnología, con la tecnología.

13. Isabel: para que tenga menos labores que hacer

14. Profesor: Sí, ¿es aplicable lo que dice esta información de Perú 21 a todas las madres del Perú?

16. Estudiantes: no, no, no (en coro) 
17. Profesor: ¿ ¿a todas las madres del Perú?

18. Estudiantes: no, no, no es aplicable

19. Profesor: no es aplicable, ¿por qué?

20. Isabel: no, porque las que viven en la sierra no hay tanta tecnología

21. Profesor: ya

22. Isabel: $\quad y$ además a algunas madres no les gusta estar con eso, con el celular

23. Andrea: algunas madres no tienen tanta economía para estar con la tecnología

24. Profesor: ya, esta información, entonces, si no apunta a todas las madres del Perú, ¿a qué tipo de madres apunta?, ¿a quiénes?

26. Ana: a las madres con dinero

27. Profesor: a las madres con dinero [...]. (Interacción maestro-estudiantes [el énfasis es mío]).

En este extracto, a partir de la pregunta “¿cuál es la característica que presentan aquí para la mamá moderna?", el maestro plantea la necesidad de pensar la visión particular que proyecta el texto. El uso del adverbio "aquí" da cuenta de la situacionalidad del discurso. Esto implica que, en otros textos, la forma como es tratado este tema puede ser diferente. Si bien en los siguientes turnos los participantes solo recuperan información literal, en la segunda y tercera preguntas que plantea el profesor - “ ¿es aplicable lo que dice esta información de Perú 21 a todas las madres del Perú??" “¿a qué tipo de madres apunta?”-, revela la necesidad de pensar en cómo los textos incluyen determinadas voces y excluyen otras (Cassany y Castellà, 2010). La pregunta planteada por el profesor podría ser parafraseada de la siguiente manera: ¿todas las madres del Perú están incluidas en la representación de mamá moderna que construye el diario?

En la respuesta de las estudiantes hay dos aspectos a notar. El primero está relacionado con la respuesta de Isabel: "en la sierra no hay tanta tecnología". En esta intervención se reproduce un imaginario sobre la sierra (Vich, 2010) que no se problematiza ni se retoma en los siguientes turnos. Esta ausencia de cuestionamiento da cuenta de cómo la percepción sobre la sierra como un espacio homogéneo, que no tiene tecnología, es parte del sentido común. El otro aspecto está relacionado con la respuesta de Andrea: "algunas madres no tienen tanta economía para estar con la tecnología". Esta intervención da cuenta de cómo desde la voz de las estudiantes aparece el cuestionamiento a ciertas representaciones en términos de desigualdades económicas. Para Andrea, "estar con la tecnología" no solo se vincula con gustos o deseos, sino fundamentalmente con un poder económico. A partir del extracto analizado, se puede evidenciar que queda pendiente aprovechar las intervenciones de las estudiantes para repensar cómo ciertos significados llegan a ser más legítimos que otros. Por ejemplo, es importante reflexionar sobre los mecanismos ideológicos que

han contribuido a construir la sierra como un espacio homogéneo carente de 
tecnología, qué consecuencias concretas trae esta representación sobre la sierra en la vida de los sujetos, qué otras representaciones más problematizadoras se pueden proponer desde los espacios escolarizados.

Para seguir reforzando la visión de los textos como una construcción parcial e interesada que tiene un impacto en la vida de los sujetos, el profesor, además de presentar los textos a leer, expone los comentarios que los lectores realizan al respecto. En una oportunidad, las estudiantes leyeron una noticia que informaba sobre cómo un cirujano plástico había dopado, violado y asesinado a una adolescente. Después de haber leído la noticia, el profesor les presentó a sus estudiantes los siguientes comentarios de los lectores, que aparecieron en la página web del diario:

\section{Comentario 1}

Lamentablemente, esta chica andaba buscando algo más que ampollas para reducir grasa. Encontró la muerte por andar en cosas de adultos.

\section{Comentario 2}

No entiendo cómo una niña de dieciséis años va sola al consultorio. ¿Qué pasa con los padres?

\section{Comentario 3}

Oye, niña no era. Ya era una joven que sabía muy bien lo que hacía.

Después de leer los comentarios online, el maestro planteó la siguiente pregunta: “ ¿a quién responsabilizan los lectores de la muerte de la adolescente?”. A continuación, presento el intercambio que se produjo a partir de esta pregunta:

1. Elena: $\quad y$ el del doctor ¿cuál es? ¿No hay comentario sobre el doctor?, ¿cuál es el comentario sobre el doctor?

3. Profesor: ahí está la pregunta. Y la responsabilidad del médico, ¿dónde está?

4. Arely: no aparece, no hay

5. Profesor: lapreguntadesucompañeraes“ ¿ydóndeapareceuncomentario en contra del médico?”. ¿Cómo ha tratado la prensa este caso?, ¿en quién recae la responsabilidad de este caso?, ¿cómo lo presenta la prensa?

8. Estudiantes: en los padres, en la chica

9. Profesor: la hacen responsable a la chica, la hacen responsable a los padres

10. Ana: $\quad$ por eso, el abogado del doctor está que se basa en eso.

11. Profesor: ah, ¿el abogado del médico se está aprovechado de eso?

12. Elena: sí, y si pagan la indemnización sale en menos tiempo

13. Ana: la justicia no es justicia 
14. Profesor: chicas, chicas, vean. Aquí, en este caso, vean el tratamiento que le ha dado la prensa. De ustedes sale la pregunta de cómo, en qué momento aparece una conclusión fuerte en contra del médico, responsabilizandoalmédico, hablandodeltipodemédicos que hay. Y no hay, pues. Lo buscamos en el texto y no hay.

Sin embargo, los comentarios van siempre en contra de los padres, de la misma adolescente que ha sufrido el peor de los tratos.

20. Elena: pobrecita

21. Profesor: ustedes creen que una chica de dieciséis años, por más que haya actuado de esa forma, ¿se merecía eso?

23. Estudiantes: nooo (en coro)

24. Profesor: no se merecía eso. Vean que es importante ver cómo la prensa presenta ciertos casos. Las personas culpan a la víctima, a sus padres, pero no al verdadero responsable. (Interacción maestro-estudiantes [el énfasis es mío])

En este extracto, después de haber conversado sobre los comentarios, Elena pide, insistentemente, un comentario que explícitamente denuncie la responsabilidad del médico: "¿no hay comentario sobre el doctor?, ¿cuál es el comentario sobre el doctor?". Señalo que esta petición es insistente, porque, en los turnos previos - que no han sido reproducidos aquí-, Elena ya había expresado hasta en tres oportunidades la misma petición. El profesor aprovecha su cuestionamiento para reiterar las preguntas centrales del diálogo: “ ¿en quién recae la responsabilidad de este caso?”, “¿cómo lo presenta la prensa?”. A través de estas preguntas, el profesor plantea a sus estudiantes una visión de los discursos de la prensa como voces que construyen una versión parcial de lo que acontece, la cual está atravesada por ideologías e intereses. Asimismo, las estudiantes pueden analizar cómo las versiones que la prensa difunde tienen un impacto en los sujetos concretos y en la construcción de la realidad.

Cuando le pregunté al docente cuál era el objetivo de presentarles a las estudiantes los comentarios de los lectores de la noticia, él señaló que ellas "tienen que ver que la prensa siempre se inclina en favor de alguien, y cuando las personas leen las noticias que publica la prensa repiten esa visión". En el caso analizado, los lectores, en cierta medida, justifican el asesinato de la adolescente, pues no cuestionan al victimario, sino el comportamiento de la adolescente o de los padres. Si bien en el ejemplo analizado se observa que los lectores, en sus comentarios, reproducen las posiciones de la prensa, esto no implica un determinismo de los discursos, puesto que los sujetos tienen la posibilidad de cuestionar o proponer versiones alternas respecto a cómo la prensa presenta lo que acontece, como se observa en la interacción del maestro con sus estudiantes.

Otra manera recurrente de leer que despliega el maestro en las interac50 I ciones con sus estudiantes es la que desarrolla la interrogación constante. En 
los extractos anteriores y en el que transcribo a continuación, se evidencia el uso de preguntas como recurso didáctico para interactuar en torno al texto. El extracto siguiente surgió después de leer un texto que narraba la historia de Juanito, un niño que había sido castigado injustamente por su padre. Según la historia, Juanito llegó a casa con calificaciones desaprobatorias y el padre asumió que eran las calificaciones de su hijo, sin percatarse de que eran sus propias calificaciones, que la maestra enviaba en función de su desempeño como padre de familia.

1. Profesor: ¿ ¿cuántos personajes hay aquí?

2. Elena: $\quad$ tres

3. Profesor: ¿tres no?, ¿quiénes son?

4. Ana: mamá, papá e hijo

5. Profesor: mamá, papá e hijo. Empecemos con el papá.

¿Qué sucede con el papá?, ¿qué pueden decir ustedes del papá?

8. Ana: que es impulsivo

9. Profesor: que es impulsivo ¿por qué se caracteriza una persona impulsiva?

11. Juana: por la forma como reacciona

12. Profesor: por la forma como reacciona, impulsivo. ¿Alguien conoce?, ¿conocen a algunos papás impulsivos?

14. Estudiantes: sí [también asienten moviendo la cabeza]

15. Profesor: ¿qué más?, ¿qué otras características encuentran en el papá? Vamos, una palabra nada más para el papá. Un papá impulsivo, ¿qué más?

18. Isabel: grosero

19. Profesor: grosero, ¿por qué piensas que es un papá grosero?

20. Isabel: porque primeramente hubiera revisado bien la boleta ¿no es cierto?, luego hubiera conversado con el niño, hubiese leído, primero. [...]. (Interacción maestro-estudiantes)

En esta interacción, se pueden observar dos fenómenos. El primero está relacionado con el carácter abierto e indirecto de los interrogativos que atenúa las peticiones planteadas por el maestro, y contribuye a que las estudiantes sientan libertad y confianza para participar. Los indeterminados "ustedes" y "alguien" contribuyen a que ninguna de las estudiantes se sienta evaluada directamente. Asimismo, el hecho de que, en sus preguntas, el profesor solicite sus opiniones y recurra a sus experiencias - ¿iconocen papás impulsivos?”, “ipor qué se caracteriza una persona impulsiva?"- brinda mayores posibilidades de que las estudiantes participen en la clase. Las preguntas abiertas e indirectas - “qué sucede con el papá?”, “¿conocen a algunos papás impulsivos?" - enunciadas por el maestro favorecen la construcción de relaciones sociales más simétricas, promueven la intervención constante de las estudiantes 
y abren la posibilidad para que estas vinculen sus experiencias personales con la información del texto.

El otro fenómeno que se observa en el extracto anterior es que el maestro retoma constantemente la participación de sus estudiantes. Esta dinámica interactiva se observa, por ejemplo, en las líneas 2-3, cuando al inicio de su turno de intervención, el profesor retoma la respuesta de Elena a la pregunta anterior. El mismo fenómeno ocurre en las líneas 4-5, 8-9, 11-12, 18-19. Cuando le pregunté al profesor la razón por la que retomaba constantemente la intervención de sus estudiantes, señaló lo siguiente:

Me permite mantener un vínculo en la interacción misma de la clase. El retomar lo que dice el estudiante permite eso, ¿no? Que el estudiante tome más confianza, tenga ese acceso a que le pueda decir cosas al profesor respecto al tema ¿no? Que pueda decir sus ideas y que el profesor las retome, lo ayuda.

A diferencia de las dinámicas interactivas en las que prevalecen instrucciones u órdenes a seguir, la interrogación constante abre la posibilidad, al maestro y a las estudiantes, de conversar sobre el texto, participar activamente en la construcción de sus aprendizajes. Sin embargo, es necesario notar que, en las interacciones analizadas, las preguntas, generalmente, son planteadas por el profesor. En ese sentido, por más democrática que intente ser la dinámica interactiva, la pregunta emitida por el profesor actúa como un campo de restricción de aparente neutralidad, en el sentido que en el mismo planteamiento de la pregunta se imprime ya el punto de vista de quien la plantea (Derrida, 1982). De este modo, "Las preguntas como dispositivos pedagógicos propician el pensamiento, reflexión y comunicación. Sin embargo, a través de ellas también se puede influir y lograr respuestas que el interrogador quiere obtener" (Zárate, 2015, p.301). Por ello, es importante que en las sesiones de aprendizaje las preguntas también provengan de parte de los estudiantes. Esta dinámica abrirá nuevos espacios de diálogo y negociación en los procesos de construcción de los conocimientos, especialmente, porque en la actualidad la práctica educativa está dominada por la "pedagogía de las respuestas" (Freire y Faundez, 2013).

\section{Conclusiones y discusión de resultados}

A partir del análisis realizado, se puede afirmar, en primer lugar, que en los procesos de construcción de política no se reproducen linealmente las versiones oficiales de la política nacional. Por el contrario, en ambos niveles de apropiación -las programaciones curriculares y las prácticas pedagógicas-, los actores educativos "no son simples seguidores de los designios de alguien por encima de ellos, sino que juegan un rol fundamental en la definición de las características de la formación impartida en cada institución" (Oliart, 2011,

52 I p. 140). Los padres, los directivos, los maestros y las estudiantes negocian en 
cada nivel curricular lo que significa para cada uno de ellos leer en los últimos grados en la escuela básica secundaria. Esta agencia en el proceso de construcción de políticas educativas nos recuerda que las escuelas deben tomar decisiones cuidadosas sobre dónde se encuentran sus prioridades políticas (Ball, Maguire y Braun, 2012). Por ello, en el marco de esta investigación, es válido plantearse las siguientes preguntas: ¿qué sujetos de política se construyen en las apropiaciones que realizan los actores educativos en esta institución?, ¿en qué medida la apropiación de estas políticas, por parte de los maestros y estudiantes, crea espacios desde los cuales se puede repensar críticamente la realidad social? y ¿qué rol desempeña esta en la transformación de las desigualdades educativas y económicas?

En el primer momento de análisis, se observó cómo los padres han sido agentes centrales en la manera como la institución educativa se ha apropiado de la política nacional (DCN). El análisis evidencia que, en la escuela, los actores educativos han alineado sus programaciones curriculares en función de los contenidos que serán evaluados en los exámenes de admisión. La presión de discursos globales externos como la mercantilización de la educación y la competencia con las escuelas privadas han permeado la construcción de una nueva subjetividad de la escuela: esta es percibida por los padres como el espacio que debe preparar a las estudiantes para ingresar a la universidad y, en el futuro, acceder a mejores condiciones de vida. Así, la forma como los actores educativos han incorporado un contenido ligado a los exámenes de admisión, en este contexto, puede entenderse como un mecanismo para resistir los embates que afectan la institucionalidad de la escuela pública, que, asediada por el fenómeno de la privatización de la educación, está en camino a ser una institución en vías de extinción (Cuenca, 2013). Esta apropiación deja de ser una mera interpretación fallida de la política nacional o una práctica de sabotaje a lo que plantea el DCN (Spillane, Reiser y Reimer, 2002). Por el contrario, da cuenta de cómo los actores locales, en tanto agentes creativos, interpretan los elementos de la política nacional; dan sentido a su experiencia en un espacio socioinstitucional que heredan; y crean, a la vez, desde sus contextos particulares, sus narrativas institucionales locales, intereses y motivaciones (Levinson, Sutton y Winstead 2009).

Como investigadora, comparto con los actores educativos su preocupación en relación con los estudios superiores como factor de movilidad social en un marco de tensión entre lo que se enseña en la escuela y lo que se evalúa en los exámenes de admisión. No obstante, ¿debe abandonar la escuela pública el encargo de ser un foro público de discusión sobre las desigualdades sociales para abocarse a la preparación de las estudiantes en pro de beneficios individuales? (Henig citado en Giroux 2003). ¿Qué riesgos trae pensar y vivir la escuela pública solo como un espacio de "preparación" para rendir un examen de admisión en un contexto en el que las ideologías de mercado regulan gran parte de las relaciones sociales de los individuos? 
En un segundo momento, vemos cómo los maestros se apropian de las programaciones curriculares y construyen políticas diferentes en torno a la lectura en la interacción con sus estudiantes: mientras que la maestra privilegia una lectura instrumental, el profesor apuesta por una lectura crítica. Recordemos que, desde una perspectiva de la lectura como una práctica situada, la forma en que los maestros y estudiantes se relacionan con los textos tiene implicancias directas en las capacidades y las destrezas que las estudiantes adquieren. Por ello, es necesario preguntarse de qué modo un constante entrenamiento en la repetición y memorización de datos léxicos contribuye a que las estudiantes se construyan como sujetos capaces de transformar de manera justa y equitativa su entorno social. En paralelo, surgen otras interrogantes: ¿en qué medida la lectura de textos que se vinculan con la experiencia cotidiana de las estudiantes?, ¿hasta qué punto la familiarización constante con preguntas que promueven el pensamiento crítico permite que las estudiantes asuman posiciones críticas con respecto a situaciones problemáticas más allá de su entorno inmediato, sobre todo, cuando están a punto de egresar de la escuela secundaria?

De este modo, el privilegio de determinadas prácticas letradas en detrimento de otras tiene consecuencias en la vida individual y social de las estudiantes. Para el caso de la maestra con sus estudiantes, estas últimas están perdiendo la posibilidad de acceder a aprendizajes más críticos que multipliquen sus visiones de su realidad inmediata, que las posicionen como agentes capaces de transformar su entorno comunal y social. Asimismo, están perdiendo la oportunidad de acercarse a géneros discursivos más próximos a la literacidad académica, los mismos que son relevantes sobre todo porque las estudiantes aspiran a incorporarse al ámbito universitario. Para el caso del maestro con sus estudiantes, si bien este incorpora textos que recogen versiones de la realidad inmediata de las estudiantes y procura que ellas asuman una posición crítica al respecto, también, ha prescindido de prácticas que permitan a las estudiantes familiarizarse con los discursos académicos: reconocer los propósitos comunicativos de estos discursos, reflexionar en el tipo de registro que exige su lectura y producción, etc. En ambos casos, la familiarización con este tipo de prácticas es importante porque las estudiantes están próximas a egresar de la Escuela Básica Regular.

A partir del análisis realizado, los maestros se constituyen como agentes centrales en el proceso de construcción de las políticas. De este modo, las maneras como enseñan a leer a sus estudiantes no constituyen solo procedimientos neutrales puramente técnico-pedagógicos. Por el contrario, estos modos se enmarcan en un conjunto de roles, normas y repertorios respecto al conocimiento, los propósitos de la escuela básica, las formas de aprendizaje, en detrimento de otras visiones o concepciones de literacidad. En los espacios escolarizados, estas prácticas adquieren especial relevancia, porque tienen un impacto en la formación del estudiante y su subjetividad, por ende, en el posi-

cionamiento de este fuera de la escuela. 


\section{Referencias bibliográficas}

Apple, M. (2002). Educar como "como Dios manda". Mercados, niveles, religión $y$ desigualdad. Barcelona: Paidós Ibérica.

Ball, S. (1993). What is policy? Texts, trajectories and toolboxes. Discourse 13(2), 10-17.

- (2003). Class strategies and the educational market. The middle classes and social advantage. London: Routledge.

- (2008). The Education debate. Bristol: Policy Press.

Ball, S., Maguire, M. y Braun, A. (2012). How schools do policy. Policy enactments secondary schools. London: Routledge.

Barton, D. y Hamilton, M. (1998). Local literacies. Reading and writing in one community. London: Routledge.

- (2004). La literacidad entendida como práctica social. En V. Zavala, M. Niño-Murcia y P. Ames (Eds.), Escritura y sociedad: Nuevas perspectivas teóricas y etnográficas (pp. 109-139). Lima: Fondo Editorial de la Pontificia Universidad Católica del Perú.

Benavides, M. y Etesse, M. (2012). Movilidad educativa intergeneracional, educación superior y movilidad social en el Perú: evidencias recientes a partir de encuestas a hogares. En R. Cuenca (Ed.), Educación superior, movilidad social e identidad (pp. 51-92). Lima: Instituto de Estudios Peruanos.

Brice Heath, S. (1983). Ways with words. Language, life and work communities and classrooms. Cambridge: Cambridge University Press.

Cameron, D. (1995). Verbal hygiene. London: Routledge.

Castanheira, M. L., Crawford, T., Dixon, C. y Green, J. (2001). Interactional ethnography: An approach to studying the social construction of literate practices. Linguistics and Education, 11(4), 353-400.

Cassany, D. y Castellà, J. (2010). Aproximaciones a la literacidad crítica. Perspectiva, 28(2), 353-374.

Cassels, D. (2013). Language policy. Basingstoke, UK: Palgrave Macmillan.

Cuenca, R. (2013). La escuela pública en lima metropolitana. ¿Una institución en extinción? Revista Peruana de Investigación Educativa, 5, 73-98.

Derrida, J. (1982). Donde comienza y como acaba un cuerpo docente. En D. Grisoni (Comp.), Políticas de la filosofía. México D.F.: Fondo de Cultura Económica.

Fairclough, N. (1992). Discourse and Social Change. Londres: Polity Press.

Fishman, J. (1979). Bilingual education, language planning and English. English Word Wide, 1(1), 11-24. 
Freire, P. y Faundez, A. (2013). Por una pedagogía de la pregunta. Crítica a una educación basada en respuestas a preguntas inexistentes. Buenos Aires: Siglo Veintiuno Editores.

Gee, J. (2005). La ideología en los discursos. Lingüística social y alfabetizaciones. Madrid: Paidós.

Giroux, H. (2003). La inocencia robada. Juventud, multinacionales y política cultural. Madrid: Morata.

González, N., Eguren, M. y De Belaunde, C. (2014). Desempeño docente y aprendizaje: una aproximación a las prácticas pedagógicas del maestro peruano. Economía y Sociedad, 84, 14-20.

Halliday, M. (1985). An Introduction to Functional Grammar. London: Arnold.

Hurtado, L. (2006). Acerca de la preparación preuniversitaria. Revista del Instituto de Investigaciones Educativas, 10(17), 167-174.

Inga, M. (2007). Estrategias metacognitivas para la comprensión y producción de textos continuos. Investigación Educativa, 11(20), 45-59.

Levinson, B., Sutton, M. y Winstead, T. (2009). Education policy as a practice of power: Theoretical tools, ethnographic methods, democratic options. Educational Policy, 23(6), 767-795.

Levinson, B. y Sutton, M. (2001). Introduction: Policy as/in practice a sociocultural approach to the study of educational policy. En B. Levinson y M. Sutton (Eds.), Policy as practice: Toward a sociocultural analysis of educational policy (pp. 1-22). London: Ablex Publishing.

Mesía, Y. (2014). Construcción simbólica de grupos y jerarquías sociales. Un estudio comparativo de las ideologías lingüísticas de estudiantes de un colegio estatal y uno particular de Lima (tesis de maestría en Lingüística). Lima: Pontifica Universidad Católica del Perú, Escuela de Posgrado. Recuperado de https://bit.ly/2LPduMu

Menken, K. y García, O. (Eds.). (2010). Negotiating Language Policies in Schools: Educators as policymakers. New York: Routledge.

Oliart, P. (2011). Políticas educativas y la cultura del sistema escolar en el Perú. Lima: Instituto de Estudios Peruanos / Tarea.

Pinzas, J. (2004). Metacognición y lectura. Lima: Pontificia Universidad Católica del Perú.

Pinzas, J., Chiuyare, M. y Macavilca, K. (2008). ¿Dónde está el error?: Ejercicios de detección de errores para mejorar la comprensión de lectura: 5to grado y 6to grado. Lima: San Marcos.

Sacristan, J. (2013). En busca del sentido de la educación. Madrid: Morata.

Sanz, P. (2015). El tránsito de la escuela pública a la escuela privada en el sector emergente de Lima Metropolitana: ¿Buscando una mejor calidad? Revista Peruana de Investigación Educativa, 7, 95-125. 
Schleppegrell, M. (2005). The language of schooling. A funcional linguistics perspective. London: Routledge.

Scribner, S. y Cole, M. (1981). The psychology of literacy. Cambridge: Harvard University Press.

Spillane, J., Reiser, B. y Reimer T. (2002). Policy implementation and cognition: Reframing and refocusing policy implementation research. Review of Educational Research, 72, 387-431.

Street, B. (1984). Literacy in theory and practice. New York: Cambridge University Press.

(2004). Los nuevos estudios de literacidad. En V. Zavala, M. Niño-Murcia y P. Ames (Eds.), Escritura y sociedad: Nuevas perspectivas teóricas y etnográficas (pp. 81-107). Lima: Fondo Editorial de la Pontificia Universidad Católica del Perú.

Torres, J. (2002). Educación en tiempos de neoliberalismo. Madrid: Morata. - (2010). La justicia curricular. El caballo de Troya de la cultura escolar. Madrid: Morata.

Tubino, F. (2007). Las ambivalencias de las acciones afirmativas. En J. Ansión y F. Tubino (Eds.), Educar en ciudadanía intercultural (pp. 91-110). Lima: Fondo Editorial de la Pontificia Universidad Católica del Perú.

Unamuno, V. (2015). Los hacedores de la EIB: un acercamiento a las políticas lingüístico-educativas desde las aulas bilingües del Chaco. Archivos Analíticos de Políticas Educativas, 23(101). Recuperado de http://dx.doi. org/10.14507/epaa.v23.2061

Young, M. (1999). Multifocal educational policy research: Toward a method for enhancing traditional policy analysis. American Educational Research Journal, 36, 677-714.

Zárate, A. (2015). El uso de las preguntas de comprensión crítica en los libros de texto. Foro de educación, 13(19), 297-326. Recuperado de https://bit. ly/2kp7Zax

Zavala, V. (2002). Desencuentros con la escritura: escuela y comunidad en los andes peruanos. Lima: Red para el desarrollo de las ciencias sociales en el Perú.

(2012). Dilemas ideológicos en torno a la educación intercultural bilingüe: el caso de la lectura en quechua. Revista Peruana de Investigación Educativa, 4, 77-104.

Zavala, V., Niño-Murcia, M. y Ames, P. (Eds.). (2004). Escritura y sociedad. Nuevas perspectivas teóricas y etnográficas. Lima: Red para el Desarrollo de las Ciencias Sociales en el Perú.

Vich, V. (2010). El discurso sobre la sierra del Perú: la fantasía del atraso. En J. Ortega (Ed.), Nuevos hispanismos interdisciplinarios y transatlánticos. Madrid: Iberoamericana Vervuert. 


\section{Nota biográfica}

Johanna Ramírez Fernández

Es magíster en Lingüística por la Pontificia Universidad Católica del Perú y licenciada en Educación por la Universidad Nacional Pedro Ruiz Gallo de Lambayeque. Es profesora de la Pontificia Universidad Católica del Perú y de la Universidad Peruana de Ciencias Aplicadas. Es miembro del grupo de investigación Lenguaje en Sociedad y del Grupo de Investigación en Lenguaje, Cultura y Educación. Sus intereses están centrados en el análisis de las prácticas letradas académicas y en el análisis crítico de los discursos de la prensa. 